\title{
Resistance training for hot flushes in postmenopausal women: Randomized controlled trial protocol
}

Emilia Berin, Mats Hammar, Hanna Lindblom, Lotta Lindh Åstrand and Anna-Clara Spetz

\author{
Linköping University Post Print
}

\section{Tweet}

N.B.: When citing this work, cite the original article.

Original Publication:

Emilia Berin, Mats Hammar, Hanna Lindblom, Lotta Lindh Åstrand and Anna-Clara Spetz, Resistance training for hot flushes in postmenopausal women: Randomized controlled trial protocol, 2016, Maturitas, (85), 96-103.

http://dx.doi.org/10.1016/j.maturitas.2015.12.015

Copyright: Elsevier

http://www.elsevier.com/

Postprint available at: Linköping University Electronic Press

http://urn.kb.se/resolve?urn=urn:nbn:se:liu:diva-126250 


\section{Resistance training for hot flushes in postmenopausal women: randomised controlled trial protocol}

Short title: Resistance training for hot flushes

Emilia Berin, medical student, 1 , Mats L. Hammar, MD, $\mathrm{PhD}_{1}$, Hanna Lindblom, $\mathrm{PT}, \mathrm{PhD}$ student2, Lotta Lindh-Åstrand, RN, PhD 1 , Anna-Clara E. Spetz Holm, MD, PhD 1

${ }^{1}$ Obstetrics and Gynecology, Department of Clinical and Experimental Medicine, Faculty of Medicine and Health Sciences, Linköping University, SE-581 83 Linköping, Sweden 2Division of Physiotherapy, Department of Medical and Health Sciences, Faculty of Medicine and Health Sciences, Linköping University, SE- 58183 Linköping, Sweden

Corresponding author: Anna-Clara E. Spetz Holm, MD, PhD, Obstetrics and Gynecology, Department of Clinical and Experimental Medicine, Faculty of Medicine and Health Sciences, Linköping University, Linköping SE-581 83, Sweden. E-mail: annaclara.spetz.holm@liu.se. Fax number: +46101033194. 


\begin{abstract}
Objectives

Hot flushes and night sweats affect $75 \%$ of all women after menopause and is a common reason for decreased quality of life in mid-aged women. Hormone therapy is effective in ameliorating symptoms but cannot be used by all women due to contraindications and side effects. Engagement in regular exercise is associated with fewer hot flushes in observational studies, but aerobic exercise has not proven effective in randomised controlled trials. It remains to be determined whether resistance training is effective in reducing hot flushes and improves quality of life in symptomatic postmenopausal women. The aim of this study is to investigate the effect of standardised resistance training on hot flushes and other health parameters in postmenopausal women.
\end{abstract}

\title{
Study design
}

This is an open, parallel-group, randomised controlled intervention study conducted in Linköping, Sweden. Sixty symptomatic and sedentary postmenopausal women with a mean of at least four moderate to severe hot flushes per day or 28 per week will be randomised to an exercise intervention or unchanged physical activity (control group). The intervention consists of 15 weeks of standardised resistance training performed three times a week under supervision of a physiotherapist.

\section{Main outcome measures}

The primary outcome is hot flush frequency assessed by self-reported hot flush diaries, and the difference in change from baseline to week 15 will be compared between the intervention group and the control group.

\section{Conclusion}

The intention is that this trial will contribute to the evidence base regarding effective treatment for hot flushes.

Key Words: Menopause; Hot flashes; Resistance training; Vasomotor symptoms; Exercise; Study protocol

Clinical trial registration: URL: www.clinicaltrials.gov; ID: NCT01987778. 


\section{Introduction}

Menopause is often associated with bothersome hot flushes, urogenital problems and sleep disturbances that may affect wellbeing and reduce quality of life. Of these symptoms, hot flushes is the most prominent, occurring in around 75\% of all women.[1] The median duration of hot flushes has been reported to be 4-5 years, [2] but can occur for more than 10 years after menopause and may also be life-long.[3]

Hormone therapy (HT) with oestrogen alone or combined with progestogens is the most effective treatment, decreasing the frequency of hot flushes by at least 75\%.[4] However, usage has declined[5] since the publication of trials associating HT with cardiovascular events and breast cancer.[6] Today, HT is only recommended for moderate and severe hot flushes in the lowest dose possible, for the shortest possible period of time.[7] As a result, there is a need for alternative treatments.

\subsection{Physical exercise}

Exercise has been proposed as an optional treatment, but studies of the association between physical activity and hot flushes have so far been inconclusive.[8]

Observational data have suggested that regular physical activity is associated with fewer hot flushes and a shorter duration of symptoms.[2, 9-11] On the other hand, other studies have failed to show such an interaction, $[12,13]$ or have even reported an increased risk of hot flushes among the most physically active women.[14]

One mechanism through which oestrogen is thought to act to prevent hot flushes is through the induction of hypothalamic $\beta$-endorphin. $\beta$-endorphin is an endogenous opioid that may stabilize the thermoregulatory centre, possibly by decreasing the thermal sensitivity of preoptic hypothalamic neurons.[15] After menopause, central $\beta$ endorphin levels decrease[16] but their production can be stimulated with oestrogen treatment[17] and is also believed to be induced by exercise.[18, 19]

The randomised controlled trials (RCT) that exist [20-24] have generally been unable to show a significant decrease in hot flushes after an aerobic exercise intervention. A recent Cochrane review concluded that there is still insufficient evidence to determine whether exercise reduces vasomotor symptoms in postmenopausal women and that good-quality trials are needed.[8] The European Menopause and Andropause Society drew the same conclusion in their position statement on non-hormonal management of menopausal vasomotor symptoms.[25] Previous studies have struggled with poor compliance in some groups $[20,23,24]$ and used a wide variety of exercise modalities and intensities, which could explain the lack of significant treatment effect. Likewise, in the newly published position statement by the North American Menopause Society, aerobic exercise is not recommended for vasomotor symptoms.[26]

Physical exercise is implicated in disease prevention in numerous other ways. After menopause, the fat distribution in women tends to shift, with increasing accumulation of visceral adipose tissue.[27] Visceral adiposity in turn, is associated with chronic lowgrade inflammation and thereby an increased risk of diseases such as type-2 diabetes mellitus, dyslipidaemia, several forms of cancer and cardiovascular disease.[28, 29] Physical exercise on the other hand, has been related to an anti-inflammatory state and lower levels of pro-inflammatory cytokines in both observational and intervention studies.[30] Moreover, exercise may induce a browning of white adipose tissue, 
generating a more favourable metabolic state.[31] In contrast to white adipose tissue, brown adipose tissue mainly produces heat and has been inversely related to obesity and type- 2 diabetes mellitus. [32]

Physical activity is also thought to affect the length of telomeres; repetitive sequences of DNA at the end of chromosomes that contribute to the stability of the genome. The telomeres shorten as a normal part of aging, but telomere attrition has also been associated with cardiovascular disease, oxidative stress and inflammation.[33] Physical activity may prevent telomere attrition, and postmenopausal women engaging in regular exercise had longer telomeres, although rigid evidence is lacking.[34]

In summary, exercise is an appealing intervention because of the established and probable health effects it may bring in addition to a possible treatment effect on hot flushes. Aerobic exercise has been the focus of several previous RCT:s but to our knowledge, this is the first trial designed to study resistance training (RT) as a treatment for hot flushes. The hypothesis is that RT will reduce hot flushes and positively affect a number of health parameters in postmenopausal women based on the studies presented above.

\subsection{Aims}

The aim is to investigate the effect of standardised RT on hot flushes and other health parameters in postmenopausal women. Specifically, we intend to test the hypothesis that 15 weeks of standardised RT reduces the frequency of moderate and severe hot flushes in symptomatic, sedentary, postmenopausal women more than in a sedentary control group asked not to change exercise habits over 15 weeks. We here present detailed information on the design of the study, intervention and outcome measures.

\section{Methods}

\subsection{Trial design}

This is an open, parallel-group, randomised controlled trial with participants individually randomised to intervention or a control group (no treatment) in a 1:1 allocation. The trial is conducted in Linköping, Sweden.

\subsection{Study Population}

We intend to include postmenopausal women with natural or induced menopause who experience a minimum of four moderate to severe hot flushes per day or 28 per week over a two week long screening period. To ensure that we include only sedentary women, we restricted the total weekly time allowed spent on physical activity adapted from the recommendations on physical activity by the World Health Organization.[35] Detailed eligibility criteria are presented in Table 1. 
Table 1. Inclusion and exclusion criteria

\begin{tabular}{|c|c|}
\hline on criteria & $\begin{array}{l}\text { - Postmenopausal women, defined as } \\
-\quad \geq 12 \text { months since the last natural menstrual period } \\
\text { - } \text { Hysterectomy and FSH }>20 \mathrm{mIU} / \mathrm{ml} \\
\text { - } \text { Amenorrhea due to intrauterine device including } \\
\text { progestogen and FSH }>20 \mathrm{mIU} / \mathrm{ml} \\
\text { - } \text { Induced menopause after bilateral oophorectomy } \\
\text { - Induced menopause by chemotherapy or radiation } \\
\text { - } \quad \text { and amenorrhea for at least two years } \\
\text { - during a screening period of } 2 \text { weeks } \\
\text { - G45 years } \\
\text { - } \text { structured general health and physical ability to participate in } \\
\text { Able to understand and communicate in Swedish orally and } \\
\text { in writing } \\
\text { Given signed informed consent to participate in the study }\end{array}$ \\
\hline Exclusion criteria & $\begin{array}{l}\text { - }>75 \text { minutes per week of moderate- to vigorous-intensity } \\
\text { physical activity. } \\
\text { - } \quad \text { b } 25 \text { minutes per week of physical activity of any intensity } \\
\text { - } \quad \text { Systolic hemoglobin }<110 \mathrm{~g} / \mathrm{l} \\
\text { pressure }>100 \mathrm{mmHg} \text {. } \\
\text { - Use of systemic HT for menopausal symptoms during the last } \\
2 \text { months. Local estrogen treatment for vaginal atrophy is } \\
\text { allowed, as is per oral estriol treatment. } \\
\text { - Use of selective serotonin and/or noradrenalin reuptake } \\
\text { inhibitors or other substances, including natural remedies, } \\
\text { with a possible effect on vasomotor symptoms. Subjects will } \\
\text { not be excluded if the treatment is deemed stable and } \\
\text { continuous and has no relation in time to the onset of flushes. } \\
\text { - Medical condition under investigation or unstable condition } \\
\text { that could affect hot flushes, like thyroid disease. } \\
\text { - Any medical condition limiting the ability to participate fully } \\
\text { in RT, like active fibromyalgia. }\end{array}$ \\
\hline \multicolumn{2}{|c|}{$\begin{array}{l}\text { FSH - follicular stimulating hormone; RT - resistance training; HT - hormone therapy. } \\
\text { aMild hot flush - sensation of heat without sweating. Moderate hot flush - sensation of } \\
\text { heat with sweating. Able to continue activity. Severe hot flush - sensation of heat with } \\
\text { sweating. Need to pause activity (to shower, remove/change clothes, open a window } \\
\text { e.g.), including waking up during the night.[37] } \\
\text { bModerate intensity - requires a moderate amount of effort and increases heart rate } \\
\text { noticeably, like brisk walking. Vigorous intensity - requires a large amount of effort and } \\
\text { increases heart and respiratory rate substantially, like running.[35] }\end{array}$} \\
\hline
\end{tabular}




\subsubsection{Recruitment}

Participants are recruited by advertisements in the local newspaper and social media (Facebook), and through information posters displayed in outpatient departments at Linköping University Hospital. Interested women are asked to respond to the adverts by e-mail or regular mail and are subsequently contacted by telephone by a research nurse or a member of the research group.

Those eligible after telephone screening are sent written information about the study and invited to a first visit where inclusion and exclusion criteria are checked. This visit includes signing an informed consent form after information and explanation of the study, a physical examination and measurements of height, weight, waist circumference, sagittal abdominal diameter, blood pressure and capillary haemoglobin. Finally, all women eligible after visit 1 receive a diary to register hot flushes and physical activity for a screening period of 14 days.

After two weeks, the women attend a second visit where a final evaluation of eligibility is done based on the number of hot flushes recorded in the screening diary. During visit 2 , the definite decision of inclusion is made, and included participants are randomised to either 15 weeks of standardised RT (intervention group) or no treatment (control group). They receive hot flush diaries to register symptoms continuously throughout the study period, and instructions on how to fill them in correctly. Baseline questionnaires regarding health-related quality of life and physical activity are completed and baseline blood samples for analysis of secondary outcome variables are drawn. These are defined in detail below.

At 15 weeks, all participants attend a final visit where baseline measurements and tests are repeated. An MRI scan is performed on both groups at baseline and at 15 weeks for analysis of muscle mass and white and brown adipose tissue. The choice to participate in MRI scanning is optional and does not affect recruitment. An overview of the study design is presented in Figure 1. 


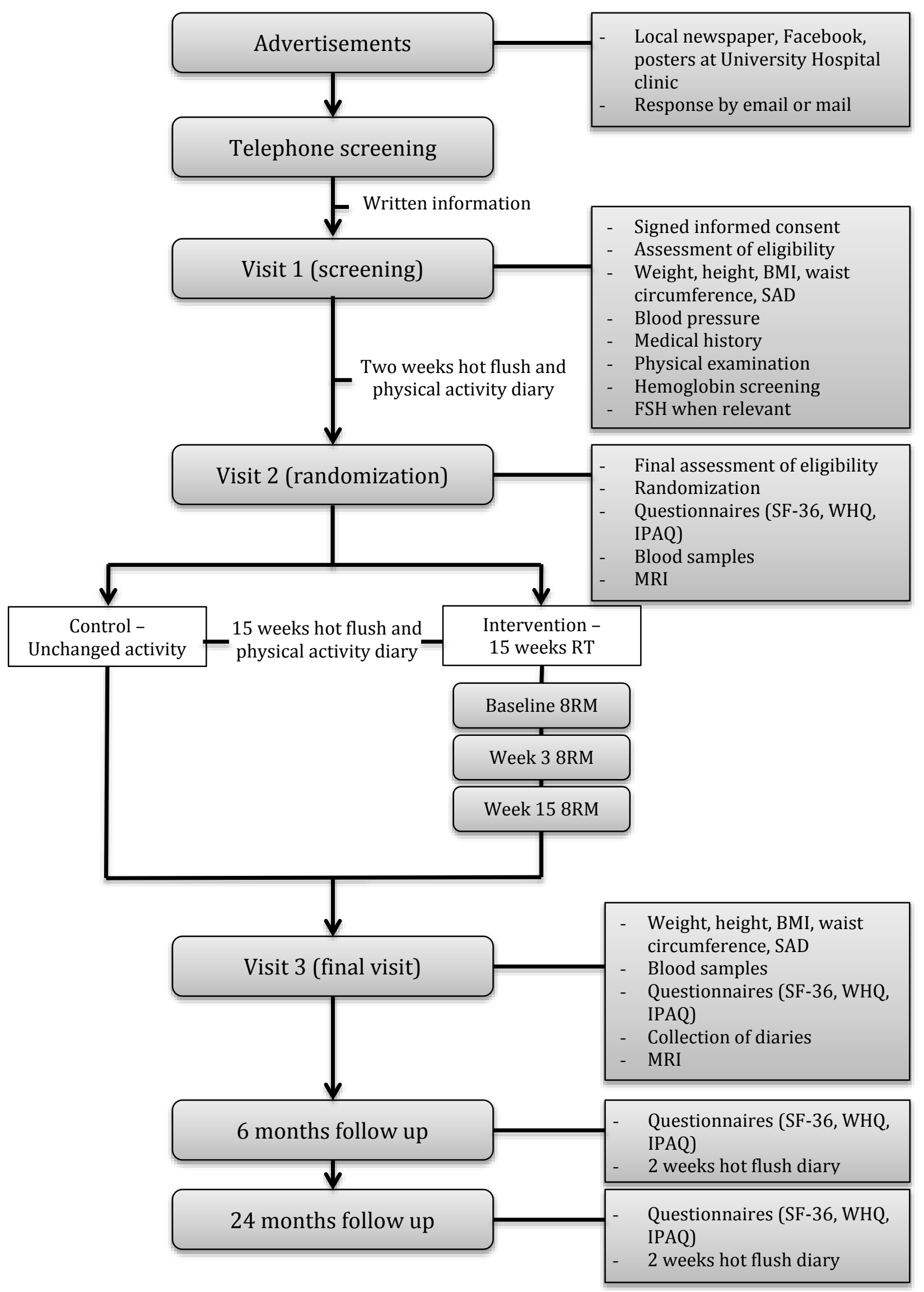

Figure 1. The flow of participants in the study, including outcome measures and follow-up points. BMI=body mass index; $\mathrm{SAD}=$ sagittal abdominal diameter; $\mathrm{FSH}=$ follicular stimulating hormone; SF-36=Short Form 36; WHQ=Women's Health Questionnaire; IPAQ=International Physical Activity Questionnaire; MRI=magnetic resonance imaging 


\subsection{Intervention}

The intervention consists of 15 weeks of structured, standardised RT performed three times per week. The training takes place at a local gym facility easily accessible by private and public transport and is monitored by an experienced physiotherapist (HL). Each participant receives an individual introductory session with the physiotherapist to familiarise with the exercises and find the correct settings on the resistance machines.

\subsubsection{Resistance training program}

All participants in the intervention group follow the same RT program, developed to target all major muscle groups and be technically easy and safe to perform. It is constructed in line with the American College of Sports Medicine (ACSM) guidelines on RT for novices[36] and focuses on improving strength and muscle mass with a gradual increase in loads. The program contains eight exercises, of which six are performed in seated resistance machines and two are body-weight exercises.

The exercises are as follows: leg press, chest press, seated row, leg extension, leg curl, lat pull-down, crunches and back raises. In accordance with ACSM recommendations,[36] the program primarily focuses on multiple-joint exercises. Every training session includes 10 minutes of warm-up on a cardio machine and dynamic and static stretching post-workout. A maximum of two exercises are allowed to be replaced by corresponding exercises after individual evaluation by the physiotherapist. If requested by the participants, additional exercises can be added to the standardised program.

Participants are free to train independently at any time during the gym's opening hours (usually 6.30AM-9PM). However, one session per week with the physiotherapist is mandatory to follow up and progress the training program, as well as to control for any health problems and adverse events. The combination of flexible training hours and regular contact with the physiotherapist was planned to increase compliance with the intervention. During every training session, participants document completed exercises, loads and repetitions in a personal logbook at the gym. To evaluate compliance, data from the logbooks and from the gym's electronic card registration system will be used.

Subjects in the intervention group are permitted to perform additional physical activity apart from the RT. However, they may not substitute a RT session for another mode of exercise and must register all additional physical activity in their hot flush diary.

Exercises are performed with 8-12 repetitions for two sets, with approximately two minutes' rest between sets. Loads are set to correspond to 8-12 repetition maximum (812RM)[36] and participants are instructed to increase the load of an exercise when they can perform more than the prescribed number of repetitions. The two body-weight exercises are performed with 15-20 repetitions per set.

During the first three weeks of training, all exercises are performed with 15-20 repetitions with loads corresponding to 15-20RM. The purpose of this introductory phase is to provide the participants with sufficient time to learn the correct technique before increasing loads, and thereby minimising the risk of injuries. Also, in a previous trial we found that many women dropped out due to a too rapid increase in physical activity.[24] Another purpose of the introductory phase was therefore to reduce dropout. 


\subsection{Control group}

Participants randomised to the control group receive instructions to not change their exercise habits or level of physical activity during the study and to not start any treatment for hot flushes. After 15 weeks, they are offered advice on physical activity, an individual gym instruction by the same physiotherapist that monitors the intervention (HL) and a free four-month membership at the gym where the training in the study takes place.

\section{Outcomes}

\subsection{Primary outcome}

The primary outcome is change in hot flush frequency (moderate and severe hot flushes) from baseline to week 15, assessed by personal pen-and-paper hot flush diaries. In the diaries, the participants daily note the number of mild, moderate and severe hot flushes experienced, and are encouraged to register continuously throughout the day to minimise recall bias. To facilitate comparison with previous studies on treatments for hot flushes, we use the criteria for symptom severity as recommended by the US Food and Drug Administration for substance trials, as seen in Table 1.[37]

\subsection{Secondary outcomes}

Secondary outcomes include:

- Health-related Quality of Life (HRQoL), assessed by Short Form 36 Health Survey (SF-36) and Women's Health Questionnaire (WHQ).

- Physical activity level, evaluated with the short form of International Physical Activity Questionnaire (IPAQ)

- Severity of hot flushes, assessed by hot flush diaries

- Muscle strength, tested using eight-repetition maximum tests at baseline, week 3 and 15 in the intervention group

- Body mass index and sagittal abdominal diameter

- Blood pressure

- Muscle volume and volume of white and brown adipose tissue using magnetic resonance imaging (MRI)

- Blood lipids and iron status (low-density lipoprotein, high-density lipoprotein, apolipoprotein-A1 and -B, ferritin, s-iron, transferrin)

- Adipo- and myokines and inflammatory markers (leptin, irisin, interleukin-4, -6, -7, $8,-10,-15$, c-reactive protein, tumor necrosis factor, brain-derived neurotrophic factor, matrix metalloproteinase-2 and-9) analysed using a multiplex magnetic bead-based assay and enzyme-linked immunosorbent assay in plasma and after white blood cell stimulation.

- Telomere length in DNA isolated from blood samples using a real-time PCR-based method.[38]

\subsubsection{Quality of life}

We intend to evaluate the effect of RT on HRQoL with two different questionnaires at baseline, 15 weeks, 6-months follow up and 24-months follow up. SF-36 will be used as a generic measure of HRQoL. It consists of 36 questions generating scores on eight scales related to health: physical functioning, physical role functioning, bodily pain, general health perceptions, vitality, social functioning, emotional role functioning, and mental health. For each scale, a score from 0 to 100 is calculated, with 0 representing 
maximum disability and 100 no disability. Summary measures for physical health and mental health are calculated as well.[39] SF-36 is one of the most used generic measures of health-related quality of life worldwide and the Swedish translation has been found to be valid and reliable.[40]

Women's Health Questionnaire is a 36-item questionnaire developed specifically to assess mid-aged women's emotional and physical health. Each item is answered on a four-point scale, and the points are calculated into ratings in the following domains: depressed mood, somatic symptoms, anxiety/fears, vasomotor symptoms, sleep problems, sexual behaviour, menstrual symptoms, memory/concentration and attractiveness.[41] It is available in several languages, and the Swedish version has shown good reliability and been validated for assessment of health-related quality of life in postmenopausal women.[42] The WHQ is also sensitive to change in HRQoL after treatment of hot flushes[41] and has been used in trials similar to the present, to assess change in quality of life after an exercise intervention.[22, 24]

\subsubsection{Physical activity level}

Physical activity level will be assessed using the short, last 7-day form of IPAQ. It contains 9 questions regarding the amount of time spent on vigorous-intensity activity, moderate-intensity activity, walking and sitting during the last seven days. IPAQ is widely used and the most extensively validity-tested physical activity questionnaire available.[43]

All participants also register physical activity habits in their hot flush diaries. There, they daily note minutes spent on any physical activity lasting more than 10 minutes. If no physical activity is performed on a certain day, it is indicated by writing "no" or " 0 " in the empty field. Data from the logbooks and from the gym's electronic card registration system will also be used to assess participation in training sessions.

\subsubsection{Muscle strength}

Muscle strength will be evaluated in the intervention group using the 8RM test at baseline, 3 weeks and 15 weeks. The test defines the maximum resistance with which an individual can perform an exercise for eight repetitions with a correct technique. A study of healthy adults reported a test-retest correlation coefficient of 0.99 in total for the 8RM test, illustrating good reliability.[44] Another study validated the 8RM test against isokinetic dynamometry, the gold standard of measuring muscle strength, and found correlations between 0.71-0.85.[45] Moreover, the 8RM test can be integrated into the training program and used to prescribe the volumes and loads of RT.[36]

The same physiotherapist (HL) performs the test on all participants and occasions. The same exercises used in the RT program are used for the strength evaluation, except for the two body-weight exercises (crunches and back raises) that cannot be tested in the same manner. Consequently, the exercises included in the 8RM testing are the following: chest press, leg press, seated row, leg curl, lat pull-down and leg extension. As recommended, the test is preceded by 7 minutes of warm-up of the entire body and completion of several repetitions with only light resistance to obtain the correct technique. Thereafter, the resistance is increased to determine 8RM, within a maximum of five trials per exercise. All repetitions will be performed with a correct posture, full range of motion and a constant velocity. 


\subsubsection{Blood sampling}

Fasting venous blood samples are collected from all participants at baseline and at 15 weeks. These will later be analysed for inflammatory markers (specified above in 3.2), blood lipids, iron status, telomere length and myokines. In the intervention group, another blood sample is drawn within four hours of the 8RM baseline- and 15-week tests, for analysis of myokines after exercise.

\subsubsection{Magnetic Resonance Imaging}

To analyse body composition, an MRI scan (Ingenia 3.0T, Philips Healthcare) is performed on both groups at baseline and at 15 weeks. Measurements will include quantification of total, subcutaneous, visceral and intramuscular adipose tissue as well as discrimination of brown and white adipose tissue. In addition, the MRI images will be used to measure the volume of major extensor and flexor muscles.

\subsubsection{Qualitative methodology}

To obtain a deeper understanding of factors that may facilitate or impede a lifestyle change, we will conduct in-depth interviews with both compliant and non-compliant participants from the intervention group at 15 weeks, as well as with dropouts. The aim is both to explore the women's experience of adopting a change in lifestyle and to investigate the factors that influence the decision to drop out or remain in an exercise trial. The interviews will be conducted by one of the authors (EB) and an independent researcher. All interviews will be recorded, transcribed and later analysed using thematic analysis.[46]

\subsection{Sample size}

A pilot study including 16 participants with the same methodology as the present was commenced in October 2013, and recruitment has since taken place twice yearly. A sample size calculation was performed based on the primary outcome measure in the pilot study, i.e. change in mean hot flush frequency from week 0 to 15 . Results from a previous study[24] indicated that a 50\% reduction in moderate and/or severe hot flushes could be expected, which was considered a clinically significant effect. The standard deviation for difference in mean percentage change was 0.52 in the control group and 0.25 in the intervention group. With an alpha-level of 0.025 and a beta-level of 0.8 , it was calculated that 32 participants in total was needed to detect a $50 \%$ reduction in hot flushes. With an expected dropout rate of $20 \%$, we therefore need a minimum of 40 participants (20 per group). In the pilot results, hot flushes did not change at all in the control group. This was somewhat surprising since it could be expected that symptoms would decrease to some extent in both groups solely by being included in the trial. To increase the power, the goal was set to include 60 participants in total.

\subsection{Randomisation and allocation concealment}

To ensure that the intervention and control groups are equal in size, randomisation is conducted in blocks whose sizes will be kept unknown to the researchers until the end of the study. An independent statistician used a computer-based random number generator (Stata 13.1, StataCorp LP) to create the allocation sequence. It was thereafter concealed in sequentially numbered, opaque and sealed envelopes by an independent research organization affiliated with Linköping University unrelated to the recruitment of participants. 
The envelopes are stored in a locked location at the clinic and handled by a research nurse until the second visit, when the decision of inclusion in the trial is made. When a new study participant is to be enrolled, the nurse retrieves the envelope with the lowest number. After verification of eligibility, including a written informed consent, the envelope is opened to reveal group allocation.

Because of the nature of the intervention, it is not possible to blind study participants, researchers enrolling participants or the physiotherapist who will be supervising the RT in this trial. However, outcome adjudicators will be blinded to group allocation when performing the analysis for the primary outcome and for analyses of secondary outcomes whenever possible. This includes researchers and staff handling blood samples and performing the MRI scans.

\subsection{Adverse events}

Adverse events are monitored by several means. First, participants are asked about health problems and changes in their health status at every follow-up point in the study from baseline to 24 months. Second, the physiotherapist reports any health problems expressed by the participants during the training sessions, including any event or illness impeding RT. Third, all participants are asked to register any health issues in their hot flush diaries and encouraged to contact the research team when needed. In the diaries, both groups also register use of any new medications, including over-the-counter substances. Any harms related to the intervention will be reported with the results. Normal delayed-onset muscle soreness is an expected effect of the RT and will not be considered an adverse event.

\subsection{Statistical methods}

To examine the efficacy of the intervention to treat hot flushes, the change in mean hot flush frequency from baseline (the screening weeks) to week 15 will be analysed with ANOVA for repeated measures, with comparisons of the two groups. Using ANOVA also makes it possible to adjust for differences at baseline when relevant. The primary analysis of the material will be conducted according to the intention-to-treat principle; that is, all randomised participants with at least one reported value post randomisation will be included regardless of compliance with the intervention. Participants who drop out without reporting anything except the baseline values will be excluded. This approach is undertaken to avoid introduction of unnecessary bias in the analyses. [47] Depending on the amount of missing data, this will be handled by mean imputation or using a mixed-models approach to the repeated measures ANOVA.

Since this is the first trial to test RT for hot flushes, further evaluation of the treatment effect with per-protocol analysis may be performed to study the influence of compliance. A participant will be defined as compliant and thereby included in the per-protocol analysis if completing a mean of two RT sessions per week, excluding missed sessions due to illness. A maximum of 21 days will be allowed to be excluded from the evaluation of compliance.

\subsection{Ethical considerations}

The protocol for this trial has been reviewed and approved by the Regional Ethical Committee in Linköping (2013/285-31). It will be conducted in line with the World 
Medical Association Declaration of Helsinki[48] and follow applicable standards of Good Clinical Practice.

Possible risks with inclusion in the trial are related to the exercise intervention and include musculoskeletal injuries and soreness. However, we have taken measures to prevent injuries by implementing a three-week introductory phase in the training and ensuring weekly contact with the physiotherapist with the ability to adapt the program. Adverse events will nonetheless be continuously monitored as described above to detect any unexpected harms.

All participants receive detailed information about the study orally and in writing and give written informed consent before participation in the study.

\section{Discussion}

Day- and night-time hot flushes is the most common symptom associated with menopause and an important reason for reduced quality of life in postmenopausal women. Also, men treated with castration therapy for prostate cancer, and breast cancer patients treated with anti-estrogenic medication are affected to the same extent.[49] Hormone therapy is effective but associated with risks that limit the possibilities of prescription. Observational data suggest exercise is a potential treatment option but evidence from randomised controlled trials is still insufficient. [8]

Our intention was to design a high-quality trial with a clearly defined and standardised intervention to boost the evidence on the issue and to report it according to CONSORT[47] recommendations. Resistance training has already been shown to prevent bone loss [50] and increase muscle strength in postmenopausal women.[51] In addition, physical exercise can improve sleep, [52] blood lipid profile,[53] well-being and HRQoL.[20] A decrease in hot flushes would thus be a welcome addition to the other important health effects of exercise after menopause.

The method of self-reporting symptoms via personal diaries is commonly used in previous hot flush studies[21, 23, 24] and has been shown to be sensitive to change with few missing data in general.[54] Although it is possible to record hot flushes objectively e.g. by measuring skin conductance, subjectively reported flushes correlate highly with objective recordings in a laboratory environment.[55] Moreover, none of the available devices for ambulatory recording of hot flushes met the requirements of both validity and patient acceptability when evaluated for a previous trial.[56] A benefit with selfreporting through diaries is also the possibility to retrieve data on hot flush severity as well as frequency, which is still not possible with objective recording. Since change in hot flush severity is an important secondary outcome measure, a subjective method of assessment is necessary.

We chose to evaluate physical activity level with a validated questionnaire (IPAQ) and via personal hot flush- and physical activity diaries. An alternative could have been to ask the participants to wear accelerometers and collect objective data on physical activity. However, we were concerned about acceptability and the extra burden that constantly wearing the device might have brought on the participants during the 15 week long intervention period. Since hot flushes are registered daily in the diaries, we 
deemed it would take little extra effort to note minutes spent on physical activity simultaneously. Thereby we collect physical activity data continuously throughout the study period, and it will be possible to relate the results from the questionnaire with the actually reported values in the diaries.

\section{Conclusion}

To the best of our knowledge, this is the first trial designed to investigate the effect of standardised RT on hot flushes. If proven effective, the intervention could be applied to other patient groups frequently troubled by hot flushes where an effective treatment is currently unavailable. These include breast and prostate cancer patients for whom oestrogen or testosterone treatment is contraindicated. Hopefully, this study will contribute to filling the evidence gap of alternative treatments for hot flushes and provide women with an effective option to help alleviate symptoms and improve quality of life.

\section{Contributors}

MLH, ACSH, LLÅ and HL planned the study design and all authors participated in the production of the analysis plan. All authors participate in the acquisition of data and HL supervises the intervention. EB prepared the first version of this manuscript and all authors participated in reviewing and drafting the manuscript. All authors have seen and approved the final version.

\section{Acknowledgements}

We are grateful to Mats Fredriksson, PhD, for constructive advice on statistics.

\section{Funding}

This trial is supported by grants from The Swedish Research Council (ID: 2014-2781) and Region Östergötland, Sweden. The funding sources had no involvement in study design, data collection or in the writing of this manuscript.

\section{Conflicts of interest}

We have no conflicts of interest to report.

\section{References}

[1] Freeman EW, Sammel MD, Lin H, Gracia CR, Pien GW, Nelson DB, et al. Symptoms associated with menopausal transition and reproductive hormones in midlife women. Obstet Gynecol. 2007;110:230-40.

[2] Col NF, Guthrie JR, Politi M, Dennerstein L. Duration of vasomotor symptoms in middle-aged women: a longitudinal study. Menopause. 2009;16:453-7.

[3] Hunter MS, Gentry-Maharaj A, Ryan A, Burnell M, Lanceley A, Fraser L, et al. Prevalence, frequency and problem rating of hot flushes persist in older postmenopausal women: impact of age, body mass index, hysterectomy, hormone 
therapy use, lifestyle and mood in a cross-sectional cohort study of 10,418 British women aged 54-65. BJOG. 2012;119:40-50.

[4] Maclennan AH, Broadbent JL, Lester S, Moore V. Oral oestrogen and combined oestrogen/progestogen therapy versus placebo for hot flushes. Cochrane Database Syst Rev. 2004:CD002978.

[5] Lindh-Åstrand L, Hoffmann M, Hammar M, Spetz Holm AC. Hot flushes, hormone therapy and alternative treatments: 30 years of experience from Sweden. Climacteric. 2015;18:53-62.

[6] Rossouw JE, Anderson GL, Prentice RL, LaCroix AZ, Kooperberg C, Stefanick ML, et al. Risks and benefits of estrogen plus progestin in healthy postmenopausal women: principal results From the Women's Health Initiative randomized controlled trial. JAMA. 2002;288:321-33.

[7] North American Menopause Society. The 2012 hormone therapy position statement of: The North American Menopause Society. Menopause. 2012;19:257-71.

[8] Daley A, Stokes-Lampard H, Thomas A, MacArthur C. Exercise for vasomotor menopausal symptoms. Cochrane Database Syst Rev. 2014;11:CD006108.

[9] Ivarsson T, Spetz AC, Hammar M. Physical exercise and vasomotor symptoms in postmenopausal women. Maturitas. 1998;29:139-46.

[10] Di Donato P, Giulini NA, Bacchi Modena A, Cicchetti G, Comitini G, Gentile G, et al. Factors associated with climacteric symptoms in women around menopause attending menopause clinics in Italy. Maturitas. 2005;52:181-9.

[11] Moilanen J, Aalto AM, Hemminki E, Aro AR, Raitanen J, Luoto R. Prevalence of menopause symptoms and their association with lifestyle among Finnish middle-aged women. Maturitas. 2010;67:368-74.

[12] Guthrie JR, Smith AM, Dennerstein L, Morse C. Physical activity and the menopause experience: a cross-sectional study. Maturitas. 1994;20:71-80.

[13] Mansikkamäki K, Raitanen J, Malila N, Sarkeala T, Mannisto S, Fredman J, et al. Physical activity and menopause-related quality of life - a population-based crosssectional study. Maturitas. 2015;80:69-74.

[14] Zhang Q, Li F, Yu Y, Yu X, Sheng Q, Zhang X. Differential factors associated with hot flashes in Chinese perimenopausal and postmenopausal women. Maturitas. 2009;63:948.

[15] Rezvani AH, Heath JE. Reduced thermal sensitivity in the rabbit by beta-endorphin injection into the preoptic/anterior hypothalamus. Brain Res. 1984;292:297-302.

[16] Nappi C, Petraglia F, Gambardella A, De Masellis G, Di Carlo C, Genazzani AR, et al. Relationship between cerebrospinal fluid beta-endorphin and plasma pituitary-gonadal hormone levels in women. J Endocrinol Invest. 1990;13:149-53.

[17] Wardlaw SL, Wehrenberg WB, Ferin M, Antunes JL, Frantz AG. Effect of sex steroids on beta-endorphin in hypophyseal portal blood. J Clin Endocrinol Metab. 1982;55:87781.

[18] Hoffmann P, Terenius L, Thoren P. Cerebrospinal fluid immunoreactive betaendorphin concentration is increased by voluntary exercise in the spontaneously hypertensive rat. Regul Pept. 1990;28:233-9.

[19] Persson LO, Karlsson J, Bengtsson C, Steen B, Sullivan M. The Swedish SF-36 Health Survey II. Evaluation of clinical validity: results from population studies of elderly and women in Gothenborg. J Clin Epidemiol. 1998;51:1095-103.

[20] Elavsky S, McAuley E. Physical activity and mental health outcomes during menopause: a randomized controlled trial. Ann Behav Med. 2007;33:132-42. 
[21] Sternfeld B, Guthrie KA, Ensrud KE, LaCroix AZ, Larson JC, Dunn AL, et al. Efficacy of exercise for menopausal symptoms: a randomized controlled trial. Menopause.

2014;21:330-8.

[22] Luoto R, Moilanen J, Heinonen R, Mikkola T, Raitanen J, Tomas E, et al. Effect of aerobic training on hot flushes and quality of life--a randomized controlled trial. Ann Med. 2012;44:616-26.

[23] Daley AJ, Thomas A, Roalfe AK, Stokes-Lampard H, Coleman S, Rees M, et al. The effectiveness of exercise as treatment for vasomotor menopausal symptoms:

randomised controlled trial. BJOG. 2015;122:565-75.

[24] Lindh-Åstrand L, Nedstrand E, Wyon Y, Hammar M. Vasomotor symptoms and quality of life in previously sedentary postmenopausal women randomised to physical activity or estrogen therapy. Maturitas. 2004;48:97-105.

[25] Mintziori G, Lambrinoudaki I, Goulis DG, Ceausu I, Depypere H, Erel CT, et al. EMAS position statement: Non-hormonal management of menopausal vasomotor symptoms. Maturitas. 2015;81:410-3.

[26] North American Menopause Society. Nonhormonal management of menopauseassociated vasomotor symptoms: 2015 position statement of The North American Menopause Society. Menopause. 2015.

[27] Toth MJ. Effect of menopausal status on body composition and abdominal fat distribution. Int J Obes Relat Metab Disord. 2000;24:226-31.

[28] Booth A, Magnuson A, Foster M. Detrimental and protective fat: body fat distribution and its relation to metabolic disease. Horm Mol Biol Clin Investig. 2014;17:13-27.

[29] Doyle SL, Donohoe CL, Lysaght J, Reynolds JV. Visceral obesity, metabolic syndrome, insulin resistance and cancer. Proc Nutr Soc. 2012;71:181-9.

[30] Soares FH, de Sousa MB. Different types of physical activity on inflammatory biomarkers in women with or without metabolic disorders: a systematic review. Women Health. 2013;53:298-316.

[31] Stanford KI, Middelbeek RJ, Goodyear LJ. Exercise Effects on White Adipose Tissue: Beiging and Metabolic Adaptations. Diabetes. 2015;64:2361-8.

[32] Lidell ME, Betz MJ, Enerback S. Two types of brown adipose tissue in humans. Adipocyte. 2014;3:63-6.

[33] Fitzpatrick AL, Kronmal RA, Gardner JP, Psaty BM, Jenny NS, Tracy RP, et al. Leukocyte telomere length and cardiovascular disease in the cardiovascular health study. Am J Epidemiol. 2007;165:14-21.

[34] Kim JH, Ko JH, Lee DC, Lim I, Bang H. Habitual physical exercise has beneficial effects on telomere length in postmenopausal women. Menopause. 2012;19:1109-15.

[35] World Health Oganization. Global Recommendations on Physical Actvity for Health. Geneva: World Health Organization; 2010.

[36] American College of Sports Medicine. American College of Sports Medicine position stand. Progression models in resistance training for healthy adults. Med Sci Sports Exerc. 2009;41:687-708.

[37] U.S. Food and Drug Administration. Guidance for Industry: Estrogen and estrogen/progestin drug preducts to treat vasomotor symptoms and vulvar and vaginal atrophy symptoms - recommendations for clinical evaluation. . Rockville, MD: Division of Drug Information (HDF-240), Center for Drug Evaluation and Research. 2003.

[38] Pooley KA, Sandhu MS, Tyrer J, Shah M, Driver KE, Luben RN, et al. Telomere length in prospective and retrospective cancer case-control studies. Cancer Res. 2010;70:31706. 
[39] Ware JE, Jr., Gandek B. Overview of the SF-36 Health Survey and the International Quality of Life Assessment (IQOLA) Project. J Clin Epidemiol. 1998;51:903-12.

[40] Sullivan M, Karlsson J, Ware JE, Jr. The Swedish SF-36 Health Survey--I. Evaluation of data quality, scaling assumptions, reliability and construct validity across general populations in Sweden. Soc Sci Med. 1995;41:1349-58.

[41] Hunter MS. The Women's Health Questionnaire (WHQ): Frequently Asked Questions (FAQ). Health Qual Life Outcomes. 2003;1:41.

[42] Wiklund I, Karlberg J, Lindgren R, Sandin K, Mattsson LA. A Swedish version of the Women's Health Questionnaire. A measure of postmenopausal complaints. Acta Obstet Gynecol Scand. 1993;72:648-55.

[43] Craig CL, Marshall AL, Sjostrom M, Bauman AE, Booth ML, Ainsworth BE, et al. International physical activity questionnaire: 12-country reliability and validity. Med Sci Sports Exerc. 2003;35:1381-95.

[44] Taylor JD, Fletcher JP. Reliability of the 8-repetition maximum test in men and women. J Sci Med Sport. 2012;15:69-73.

[45] Taylor JD, Fletcher JP. Correlation between the 8-repetition maximum test and isokinetic dynamometry in the measurement of muscle strength of the knee extensors: A concurrent validity study. Physiother Theory Pract. 2013;29:335-41.

[46] Braun V, Clarke V. Using thematic analysis in psychology. Qualitative Research in Psychology. 2006;3:77-101.

[47] Moher D, Hopewell S, Schulz KF, Montori V, Gotzsche PC, Devereaux PJ, et al. CONSORT 2010 Explanation and Elaboration: Updated guidelines for reporting parallel group randomised trials. J Clin Epidemiol. 2010;63:e1-37.

[48] World Medical Association. World Medical Association Declaration of Helsinki: ethical principles for medical research involving human subjects. JAMA. 2013;310:21914.

[49] Morrow PK, Mattair DN, Hortobagyi GN. Hot flashes: a review of pathophysiology and treatment modalities. Oncologist. 2011;16:1658-64.

[50] Nelson ME, Fiatarone MA, Morganti CM, Trice I, Greenberg RA, Evans WJ. Effects of high-intensity strength training on multiple risk factors for osteoporotic fractures. A randomized controlled trial. JAMA. 1994;272:1909-14.

[51] Asikainen TM, Kukkonen-Harjula K, Miilunpalo S. Exercise for health for early postmenopausal women: a systematic review of randomised controlled trials. Sports Med. 2004;34:753-78.

[52] Mansikkamäki K, Raitanen J, Nygard CH, Heinonen R, Mikkola T, EijaTomas, et al. Sleep quality and aerobic training among menopausal women--a randomized controlled trial. Maturitas. 2012;72:339-45.

[53] Kemmler W, Lauber D, Weineck J, Hensen J, Kalender W, Engelke K. Benefits of 2 years of intense exercise on bone density, physical fitness, and blood lipids in early postmenopausal osteopenic women: results of the Erlangen Fitness Osteoporosis Prevention Study (EFOPS). Arch Intern Med. 2004;164:1084-91.

[54] Sloan JA, Loprinzi CL, Novotny PJ, Barton DL, Lavasseur BI, Windschitl H. Methodologic lessons learned from hot flash studies. J Clin Oncol. 2001;19:4280-90. [55] Sievert LL. Subjective and objective measures of hot flashes. Am J Hum Biol. 2013;25:573-80.

[56] Carpenter JS, Newton KM, Sternfeld B, Joffe H, Reed SD, Ensrud KE, et al. Laboratory and ambulatory evaluation of vasomotor symptom monitors from the Menopause Strategies Finding Lasting Answers for Symptoms and Health network. Menopause. 2012;19:664-71. 


\section{Figure and table legends}

Figure 1. The flow of participants in the study, including outcome measures and followup points. $\mathrm{BMI}=$ body mass index; $\mathrm{SAD}=$ sagittal abdominal diameter; $\mathrm{FSH}=$ follicular stimulating hormone; SF-36=Short Form 36; WHQ=Women's Health Questionnaire; IPAQ=International Physical Activity Questionnaire; MRI=magnetic resonance imaging

Table 1. Inclusion and exclusion criteria. 